\title{
Spatial span under translation: A study of reference frames
}

\author{
S. E. Avons \\ University of Essex, Colchester, England
}

\begin{abstract}
The nature of the reference frame used to remember location sequences in a computer-presented version of spatial span was investigated by moving the template (a rectangular frame enclosing nine target squares) across the screen during presentation and/or during recall. Movement of the display during presentation substantially impaired memory in comparison with a stationary display (Experiment 1). However, there was no effect of template movement during recall (Experiment 2). In Experiments 3 and 4, the template was moved through the same screen locations during presentation and recall. When the extrinsic, or screen location, of each position was repeated identically on each trial but the sequence on the template varied, learning was not facilitated (Experiment 3). When the template sequences were repeated across trials but extrinsic location varied, the sequences were rapidly learned (Experiment 4). In this version of spatial span, location sequences appear to be encoded in an intrinsic frame of reference that is based on the template. Movement of the template during encoding impairs this process, possibly because concurrent attention shifts prevent the encoding of locations. The results are discussed with respect to recent studies of positional encoding in which multiple reference frames were available.
\end{abstract}

Although widely used as a psychological assessment tool, immediate serial recall of spatial locations (referred to here as spatial span, but also known as the Corsi blocks test) is less well understood than immediate serial recall of verbal tokens (digit or word span). The latter has received considerable attention in the empirical literature, which includes many studies of factors that influence performance, such as phonological similarity between list items, irrelevant speech or other sounds, concurrent articulatory suppression, word length, temporal grouping, and lexical properties such as word frequency. This extensive empirical effort has culminated recently in a series of explicit models of verbal serial recall (see, e.g., Burgess \& Hitch, 1999; Page \& Norris, 1998).

Spatial span has been studied less extensively. A number of dual-task studies have explored the effects of various kinds of interference on spatial span and related tasks; these studies have consistently shown that spatial tapping, spatial attention shifts, and directing eye movements to irrelevant targets cause impairment (see, e.g., Lawrence, Myerson, Oonk, \& Abrams, 2001; Pearson \& Sahraie, 2003; Smyth, 1996; Smyth \& Scholey, 1994), but that repetitive movements that involve touching body parts do not (Smyth, Pearson, \& Pendleton, 1988). These interference tasks all require that attention be directed to external stimuli in the surrounding space, although the nature of this space and its relation to the encoding of spatial span have generally been ignored (see Woodin \& Allport, 1998, for one exception). Several more recent studies have investigated encoding processes in spatial span more closely by varying the parameters of spatial sequences and measuring the effects on memory. Taken together, the studies suggest that organizing principles are used to describe a path or trajectory across a sequence of locations. Simple or redundant paths are easier to encode and remember than less predictable ones (see, e.g., Kemps, 2001; Parmentier, Elford, \& Mayberry, 2005; SchumannHengsteler, Strobl, \& Zoelch, 2004). A third type of investigation has looked for grouping effects in spatial span by clustering the spatial locations and manipulating the convergence of temporal and spatial orders (see, e.g., De Lillo, 2004; Smyth \& Scholey, 1994). These studies have shown an influence of temporospatial grouping, but some results obtained with temporospatial grouping may be confounded by path length (Parmentier, Andres, Elford, \& Jones, 2006), suggesting a simpler explanation based on spatial discrimination.

All of these studies of spatial span indicate factors that influence the ability to encode a sequence of locations or movements in space and thus help us to understand the encoding processes used in spatial span. Any attempt to model spatial span must be constrained by these findings. However, a more fundamental issue concerns the nature of the spatial representation or representations used in spatial span. Every spatial representation has a reference frame based on a fixed reference location (or set of locations), which may also be linked to a coordinate system capable of specifying direction and distance in relation to these fixed locations. This article reports an initial investigation into the spatial reference frame used in spatial span. In

S. E.Avons, savons@essex.ac.uk 
clinical use, the Corsi version of spatial span is normally presented in the form of rigid cubes attached to a board (see, e.g., B. Milner, 1971), which rests on a table. In most computerized variants of the task, the board and blocks are represented by a 2-D drawing of a rectangular frame (the board) enclosing a number of irregularly positioned squares (the blocks), presented on a vertical computer screen. The drawing resembles a plan view of the Corsi apparatus and will be referred to here as the template. In both physical formats, the locations that are used to make up the spatial span sequences are stationary with respect to the observer and the environment throughout the presentation and test phases of each trial. Hence the sequence of locations could be remembered using a variety of reference frames, either singly or in combination.

One possibility is that target locations are encoded using a system of egocentric reference frames, specifying position with respect to the body axis, head, eyes, or body parts of the observer. Egocentric reference frames are useful for coordinating actions, since the movements required to interact with objects can be computed directly from egocentric information (A. D. Milner \& Goodale, 1995). On the other hand, environmental reference systems specify location with respect to features of the environment, such as the walls or furniture of a room, or, in the case of a computerized display, location in relation to the display screen. For a discussion of environmental reference systems in relation to memory for scenes, see, for example, McNamara (2003) and Sholl and Nolin (1997). A third possibility is that the reference frame is template centered, meaning that locations are specified using a reference frame intrinsic to the template. In this case, the indicated spatial locations are described with respect to a coordinate system based on the template itself and independent of the wider environmental context. For example, the rectangular frame and orientations of the squares may specify the spatial directions up, down, right, or left, and the position of each square may be specified in relation to the frame and/or the other squares using this system of coordinates.

In the present series of studies, the nature of the reference frame used in spatial span was explored using a computerized display. The manipulation used in all cases involved translating the template around the computer screen during presentation of the sequence and/or during recall. Broadly, the rationale is as follows. Since the whole template moves without any rotation or plastic transformation, descriptions in the template-centered, intrinsic frame of reference would be unchanged by translation and therefore should show little effect of movement. However, moving the template with respect to a stationary observer would change the position of spatial targets with respect to at least some egocentric reference frames, most notably the body-centered reference frame. In addition, translation of the template would affect the position of spatial targets in the environmental coordinates provided by the computer screen, or by the dimensions of the booth in which the experiments were carried out. Because we cannot distinguish between the egocentric body-centered frame and a more general allocentric environmental frame of reference, we refer to these together as extrinsic frames of reference, and we refer to the spatial position specified in this way as extrinsically encoded. In contrast, spatial positions of targets that are encoded with respect to their position on the template are described as template centered. At various points in the discussion, reference to other specific reference frames will be made.

There is an extensive literature on reference frames in spatial memory, but the implications for spatial span are not always clear. Previous studies have not involved spatial span directly, and many investigations were designed to explore quite different cognitive demands, such as the ability to navigate freely in learned spatial environments. In addition, these studies have addressed the distinction between egocentric and allocentric (viewpoint-independent) spatial representations. The precise nature of these allocentric representations has been controversial, and it is likely that the representations have varied across tasks. As indicated above, the present study can address only the imperfect distinction between extrinsic and template-centered representations. Nevertheless, the literature provides some indication of the spatial representations that may support spatial span. The following selective review will consider several paradigms that inform the present study, including (1) experimental studies of short-term memory for single locations, (2) neuropsychological studies of memory for position, and (3) studies of memory for arrays of objects in more natural environments.

\section{Short-Term Memory for Single Locations}

One group of studies has explored the reference frames used in immediate memory for single spatial locations. In these studies, memory was tested using a briefly presented target. After a short interval, participants had to indicate the position of the target (1) in total darkness (so that target location could be encoded only egocentrically), or (2) with respect to a local visible landmark that was stationary (permitting either egocentric or allocentric encoding), or (3) with respect to a landmark that was moved between presentation and test (permitting only allocentric encoding). In at least two recent studies, the pattern of pointing errors was markedly different for the egocentric versus the allocentric conditions; when either reference frame was available, the pointing errors indicated a preference for allocentric encoding (see, e.g., Diedrichsen, Werner, Schmidt, \& Trommershauser, 2004; Lemay, Bertram, $\&$ Stelmach, 2004). These results suggest that proximal spatial position is coded with respect to a landmark, if one is available, rather than with respect to the observer's position. In terms of the present study, in which spatial targets and the surrounding environment were visible, the implication is that egocentric encoding is unlikely to contribute. Either extrinsic or template-centered coding could occur, depending on which of the available visible reference frames would be used.

\section{Neuropsychological Studies of Memory for Position}

Neuropsychological studies have also explored memory for spatial location under conditions described as 
egocentric and allocentric (which here means orientation independent), often using analogues of spatial tasks devised for use with animals (see, e.g., Morris, Garrud, Rawlins, \& O'Keefe, 1982). In one typical study, participants traced a path over a touch screen mounted above a monitor showing a circular swimming pool until they located a spatial target, the hidden platform. In the egocentric condition, the platform remained fixed relative to the observer, although the landmarks moved from trial to trial. In the allocentric condition, the platform was fixed relative to the landmarks, and the observer moved around the display. The starting point for the path varied among trials. Performance was impaired in a group of right unilateral temporal lobectomized patients but only on the allocentric version of the task (Feigenbaum \& Morris, 2004). Other studies have shown similar selective sensitivity to lesions of the right medial temporal lobe in tasks requiring allocentric encoding resistant to orientation changes (see, e.g., Abrahams et al., 1999; Goldstein, Canavan, \& Polkey, 1989; Holdstock, Mayes, Cezayirli, Aggleton, \& Roberts, 1999; Holdstock et al., 2000). Hippocampal involvement was also reflected in neuroimaging studies of virtual reality versions of these tasks when navigation by allocentric representations was required (see, e.g., Bohbot, Iaria, \& Petrides, 2004; Parslow et al., 2004).

These studies have implications for spatial span, which has been developed as a neuropsychological test. Although recent data suggest temporal lobe involvement in the allocentric spatial tasks described above, temporal lobe lesions have little effect on spatial span (Corsi, 1972; Ferreira et al., 1998; Gagnon, Foster, Turcotte, \& Jongenelis, 2004; Rausch \& Ary, 1990). A possible interpretation is that the reference frame used in spatial span differs from that used in spatial navigation tasks, and indeed one proposal is that the spatial span task relies on egocentric rather than allocentric encoding (Aguirre \& D'Esposito, 1999). An explanation in terms of egocentric encoding would explain the relative insensitivity of spatial span to hippocampal damage, its sensitivity to right parietal lesions, and its association with neglect (see, e.g., Fink et al., 2003; Hasselbach-Heitzeg \& Reuter-Lorenz, 2002; Malhotra et al., 2005; Stark, Coslett, \& Saffran, 1996). However, there is currently no direct evidence for this claim, and other accounts of the dissociation exist, such as the difference between short-term and long-term memory.

The relationship between positional memory (the precise unordered recall of a set of locations within a rectangular display) and spatial span was investigated neuropsychologically by Kessels and colleagues (Kessels, de Haan, Kappelle, \& Postma, 2002; Kessels, Kappelle, de Haan, \& Postma, 2002; see Postma, Kessels, \& van Asselen, 2004, for a review). Broadly, they reported that the accuracy of positional memory correlated with spatial span. However, in some stroke patients, positional memory was impaired, whereas spatial span was within the normal range. When comparing patients with controls, patients with right hemisphere stroke showed impairment in positional memory, but spatial span was not impaired relative to controls. This suggests that spatial span is not simply sequential ordering superimposed on the spatial representation used in positional memory, which raises the possibility that different reference frames are used. However, Kessels, Postma, Kappelle, and de Haan (2000) proposed that allocentric reference frames were used in all spatial tasks. It should be noted that this positional memory task differed from spatial span with regard to the precision of spatial localization. A small impairment in the ability to reproduce a position measurably increases errors in positional memory, in which spatial tokens are absent at recall, but not necessarily in spatial span, in which the tokens are provided at recall. Thus, dissociations between these tasks may not necessarily reflect different spatial representations.

\section{Memory for Object Arrays in Natural Environments}

One recent series of studies of the position of objects in natural environments has used recall of directions from particular locations to explore reference frames encoded in memory. For example, participants learned the location of objects in a confined space from a restricted set of viewing positions. At test, they were required to indicate the locations of objects relative to the observer, given a particular imagined observer position and heading (see, e.g., Mou, McNamara, Valiquette, \& Rump, 2004; RoskosEwoldsen, McNamara, Shelton, \& Carr, 1998; Shelton \& McNamara, 2001; Sholl \& Nolin, 1997). The results of these studies show that accurately identifying relative location depends critically on the imagined orientation of the participant at test. In some cases, the imagined orientation may show an advantage if it is aligned with the original viewing direction at test (see, e.g., Roskos-Ewoldsen et al., 1998; Sholl \& Nolin, 1997). However, the optimum imagined orientation can be influenced by other factors, such as environmental reference frames provided by the walls of the room (Shelton \& McNamara, 2001) or indeed an intrinsic reference frame provided by the array of objects (Mou \& McNamara, 2002). When competing reference frames were provided, such as when items were placed on a misaligned rectangular mat in a rectangular room, the local reference frame predominated, at least when only a single view was learned (Shelton \& McNamara, 2001). McNamara and colleagues propose that spatial memory is based on an environmental reference system, which applies on both small and large scales. They also propose an egocentric reference system in which information is transient and which is used primarily to update spatial location relative to the observer during observer movement (see Sholl \& Nolin, 1997, for a related account). These studies are important because they show that a choice from the available reference frames may be made during encoding. At least in some cases, the preferred reference frame was the one most local to the objects to be located, and intrinsic reference frames were used. Both results suggest that in the present experiments, template-centered coding will be found, although the use of an egocentric reference frame is not ruled out in immediate memory tasks. 
To summarize, at present there is no direct evidence available on the coding of locations in spatial span. Experimental studies of the localization of objects within reach suggest that position is encoded with respect to any local landmarks that are available, rather than pure egocentric position. Neuropsychological studies show that spatial span can be preserved in patients with lesions that affect learning object location with respect to a set of landmarks or recalling a set of briefly presented locations. However, in neither case is it clear that the dissociations imply a difference in the way spatial locations are represented. Finally, some recent long-term memory studies argue that a choice of which reference frame to use for encoding spatial position can be made in natural environments. The heuristics by which this choice is made are not yet clear, but it is possible that local, intrinsic reference frames have priority, with clear implications for the present study.

In the present experiments, spatial span was presented on a computer screen by drawing a template consisting of nine unfilled squares, which represented the blocks, inside a rectangular frame, which represented the board. The nature of the reference frame was investigated by translating the template around the computer screen during presentation of the sequence and/or during recall. In all cases, the locations were displayed while the template was stationary, and translation movements occurred only in the intervals between the display of each location of the sequence at input or after each attempted recall of a location at output. Experiments 1 and 2 explored the effects of moving the template in this way during presentation and during recall, respectively. In the remaining experiments, the template was moved along the same trajectory during both the presentation and the recall phases, so that memory could be based on either an extrinsic frame of reference or a template-centered frame of reference. These studies investigated the extent of learning of a constant spatial sequence across trials, when the sequence was constant in the extrinsic frame of reference (Experiment 3 ) and when it was constant in the template-centered reference frame (Experiment 4).

\section{EXPERIMENT 1}

In this experiment, participants remembered spatial sequences under two conditions. In the control condition, the template remained stationary during presentation and recall. In the experimental condition, the template moved in a random direction at a speed of $10 \mathrm{~cm} / \mathrm{sec}$ during the intervals between the display of each target location. At the end of the sequence, the template remained stationary while the sequence was recalled. Moving the template from one location to another during presentation meant that the location at which the squares were displayed as specified by an extrinsic (egocentric or environmental) reference frame could not be used to recall the sequence at test. Thus, if performance on spatial span was supported by spatial memory based on one of these extrinsic frames of reference, moving the template during presentation should have impaired performance. If, on the other hand, spatial memory was template centered and local to the template, then translation of the template during presentation should not have impaired performance. A third consideration is that translating the template would require shifts of spatial attention during input, and there is good evidence that shifts of spatial attention to irrelevant stimuli impair span performance (see, e.g., Pearson \& Sahraie, 2003; Smyth \& Scholey, 1994). This is a possibility even if a template-centered representation is used; this issue will be considered later.

\section{Method}

Participants. Twenty-four undergraduate or postgraduate students at the University of Essex, 19 females and 5 males, participated in this experiment. The mean age was 22.5 years $(S D=6.4)$. Fourteen participants were paid $£ 2.50$ for their participation; the remainder took part for course credit.

Apparatus. All of the experiments reported here were displayed on Mitsubishi Diamond Plus 91 17-in. monitors controlled by a Macintosh G3 computer, using 1,024 $\times 768$ pixel resolution. Software was written in $\mathrm{C}$, incorporating the VideoToolBox subroutines (Pelli, 1997).

Materials. The template consisted of a rectangle measuring $17.5 \mathrm{~cm}$ horizontally $\times 13 \mathrm{~cm}$ vertically. Nine unfilled squares, with sides measuring $1.4 \mathrm{~cm}$, were placed within this rectangle. On each trial, the squares were positioned in a different random arrangement, with the constraint that the centers of any two squares could not be closer than $5.2 \mathrm{~cm}$, or 3 times the side length of the squares. Each spatial sequence consisted of seven squares, chosen randomly from the nine squares of the template without replacement.

Procedure. At the beginning of each trial, the template was displayed in the center of the screen for $1 \mathrm{sec}$. Following this, the first location was shown by filling one square in black for $0.5 \mathrm{sec}$, followed by a $0.5-\mathrm{sec}$ interval during which all squares were unfilled. The second location was then displayed for $0.5 \mathrm{sec}$, followed by $0.5 \mathrm{sec}$ with all squares unfilled; this cycle repeated a total of seven times. Thus, seven squares of the template were displayed in sequence at a 1-sec presentation rate. After the last cycle of the presentation sequence, there was a further 0.5 -sec retention interval, and then a tone sounded. After the tone, the participant used the computer mouse to click on the squares in the order in which they had been displayed. As each square was selected, it was filled in black and could not be selected again. To keep the recall conditions consistent with those of later experiments, after each location was chosen, the mouse cursor disappeared for $0.5 \mathrm{sec}$. When seven squares had been selected, the screen was then cleared, and the participant clicked the mouse in the bottom right corner of the screen to initiate the next trial.

In the control condition, the template remained in the center of the screen during presentation and recall. This condition conformed to a conventional computer-presented version of spatial span with a fixed sequence length. In the movement condition (Figure 1A), the template was initially displayed in the center of the screen, where the first square was displayed for $0.5 \mathrm{sec}$. During the following 0.5 -sec interval, the template then moved to a new position on the screen where the next target location was displayed, and so on for seven cycles. Thus, the template moved to a new screen position after each of the seven squares was displayed. Each movement of the template lasted $0.5 \mathrm{sec}$ at a constant speed of approximately $10 \mathrm{~cm} / \mathrm{sec}$. The direction of each movement was chosen randomly with the constraint that the whole template remained visible on the screen at all times. Thus, the template moved about $5 \mathrm{~cm}$ after each square was displayed. After this presentation sequence, the template remained stationary in its final resting position while the sequence was recalled.

Conditions were blocked and presented in counterbalanced order. Twenty trials were completed in each condition, of which the first five were discarded as practice. The experiment was preceded by a short practice session of six trials in which participants recalled a se- 

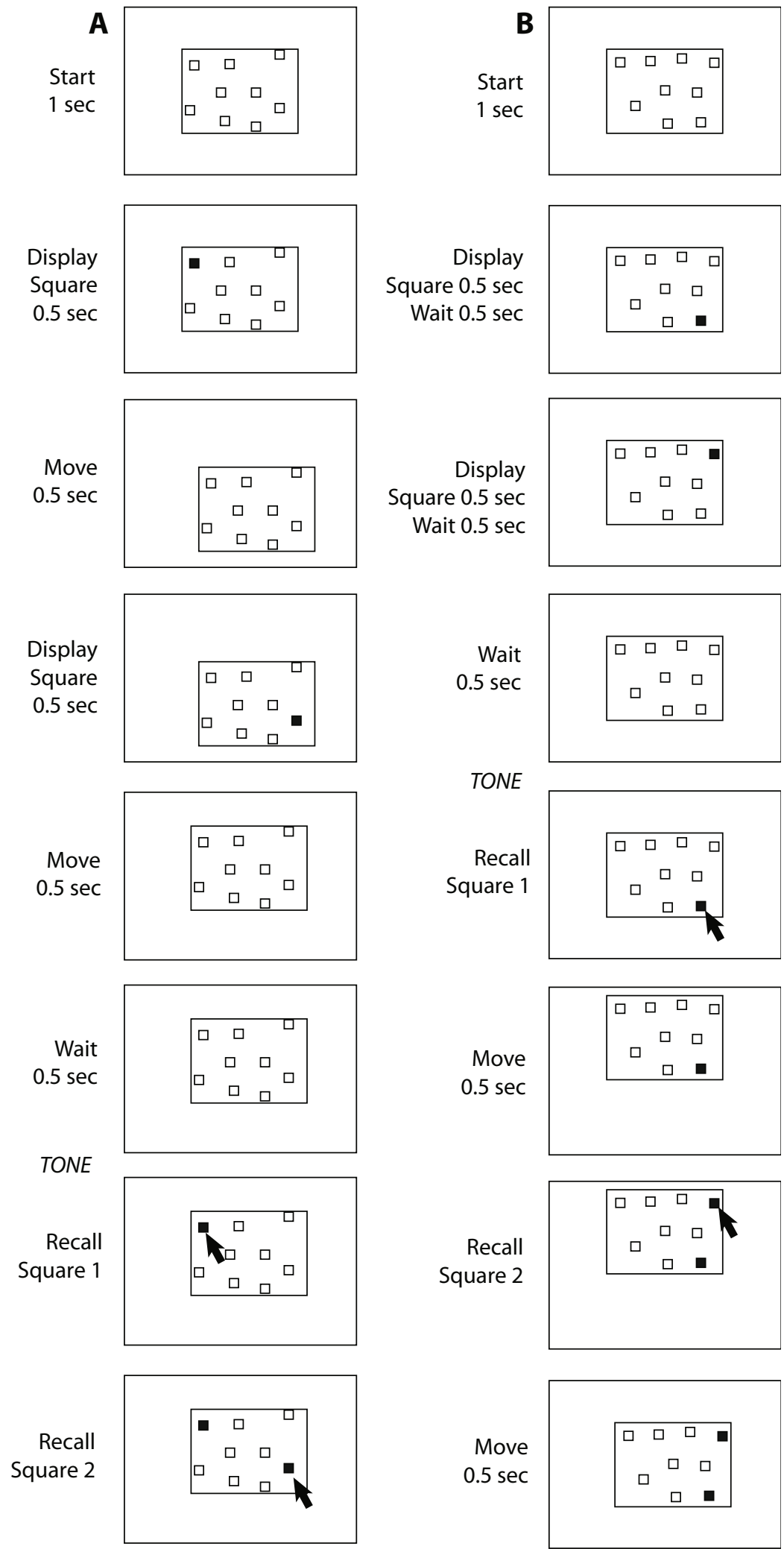

Figure 1. Illustration of the sequence of displays in the movement conditions of Experiment 1 (A) and Experiment 2 (B). Each picture shows the position of the template in relation to the border of the screen. The labels describe the display changes occurring in, and the duration of, each stage in the sequence. Pictures labeled "move" show the position of the template after the movement, which in each case lasted $0.5 \mathrm{sec}$. Bold arrows indicate the cursor position during recall. In this illustration, the sequence length is reduced to two locations. 
ries of six squares from a stationary display. Participants were seated in front of the monitor with their eyes approximately $60 \mathrm{~cm}$ from the screen. Participants were not restrained by a chinrest but were instructed to keep still during the presentation of the sequences. The experimenter remained present throughout to ensure compliance.

\section{Results and Discussion}

In Figure 2, the mean accuracy over the last 15 trials of each condition is shown in the serial position curves, which show clearly that moving the template during presentation impaired performance relative to the control condition. Moreover, the impairment occurred at all serial positions.

These observations were confirmed by a mixed $2 \times 2 \times 7$ ANOVA with order of conditions as the between-subjects factor and movement condition and serial position as the two within-subjects factors. In this and all subsequent analyses, the Greenhouse-Geisser correction was applied where sphericity was violated. The results show that there was no significant effect of the order of conditions $[F(1,22)<1.0]$, and no significant interaction of order with any other variable. As anticipated there was an effect of serial position $\left[F(3.4,73.8)=29.8, M S_{\mathrm{e}}=0.0437, p<.001\right]$. There was also a significant effect of condition $[F(1,22)=$ $\left.18.6, M S_{\mathrm{e}}=0.0338, p<.001\right]$. There was no significant interaction of condition and serial position $[F(4.0,89.0)<$ 1.0]. The same trends were observed when analyzing the proportion of trials on which all responses were correct and the sequence was reproduced perfectly. The mean proportion correct was $.22(S D=.19)$ in the static condition and $.12(S D=.12)$ in the moving condition. This difference was significant $[t(23)=4.05, p<.001]$.

These results show that moving the template during the display of a spatial sequence impaired spatial shortterm memory. The detrimental effect of movement during input on spatial span can be interpreted in one of two ways.

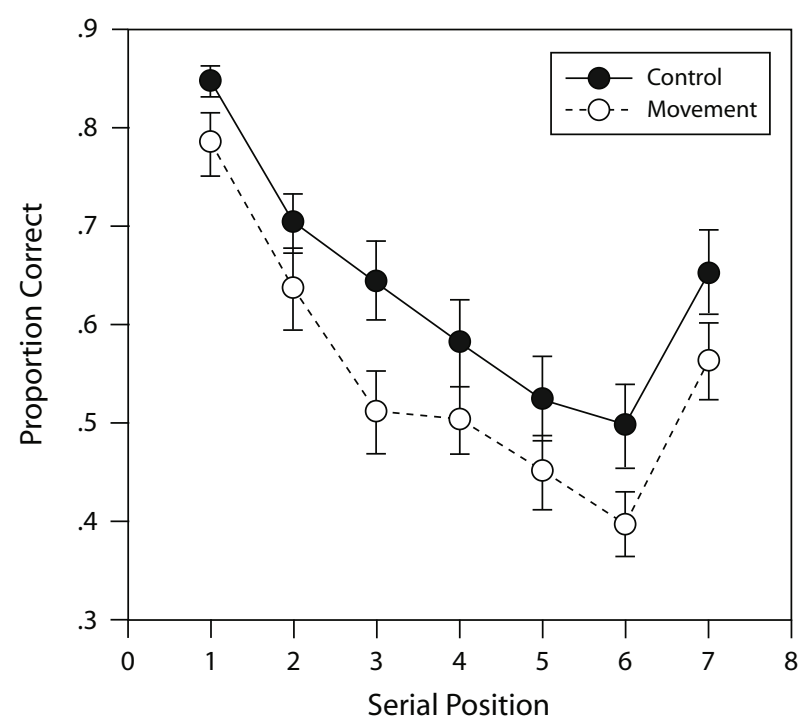

Figure 2. Accuracy in Experiment 1 of serial spatial recall in the control condition (Corsi template was stationary at presentation and during recall) and when the template moved relative to the observer during presentation.
First, from the standpoint of a contribution from extrinsic or template-centered reference frames, the results suggest that an extrinsic reference frame contributed to spatial span. Random movements of the template during sequence presentation ensured that the position of each displayed square in relation to coordinates based on the observer's body or the environment (e.g., the screen position) was (1) different from the position at test and (2) could not be used to predict position at test. Thus remembering the extrinsically encoded position of a displayed square could not have been used to recall the corresponding location at output. In contrast, in the control condition, spatial information utilizing an extrinsic reference frame was available and could have contributed to spatial span if the position of each displayed square was remembered in an extrinsic frame of reference from presentation until recall. Hence in the control condition, spatial span was potentially supported by a combination of extrinsic and template-centered spatial representations, whereas in the movement condition only the latter was available.

Another possible explanation for the impaired performance in the moving condition is that translation during input disrupted template-centered spatial encoding. Here, moving the template in unpredictable directions during presentation produced additional demands that competed with the task of encoding the spatial sequence. For example, tracking the movement of the template may have required resources otherwise used for encoding or rehearsing the spatial locations of the sequence. Previous research has shown that directing attention to irrelevant locations or making irrelevant eye movements throughout an extended retention interval impairs performance on spatial span (Pearson \& Sahraie, 2003; Smyth \& Scholey, 1994). Also, in the working memory tradition, concurrent pursuit rotor tracking and eye movements impair performance on the Brooks matrix task, which requires sequential spatial memory (for an account, see Baddeley, 1986). These interference studies imply that spatial attention mechanisms are engaged in encoding and rehearsing sequences of spatial locations. In the present study, spatial shifts of attention and pursuit eye movements were required to track the movements of the template during presentation. Although task relevant, these attention shifts may have engaged the mechanisms needed to encode the spatial sequence described on the template. To investigate this issue further, Experiment 2 was designed so that movement of the template occurred only during recall, after the spatial sequence had been encoded; in other respects, Experiment 2 was similar to Experiment 1.

\section{EXPERIMENT 2}

This experiment explored the effect of moving the template during output. Here, the control condition was identical to that of Experiment 1. In the experimental condition, the template remained at a fixed position during presentation but moved unpredictably to a new location after each square was selected during recall. The movement was of the same duration and extent as in Experiment 1.

Consider first the hypothesis that the impairment seen in the movement condition of Experiment 1 arose because 
memory for each location was encoded using an extrinsic frame of reference and that this source could not be used at the time of recall because of the template movement. If this is the explanation for the impairment of Experiment 1 , then performance should likewise be impaired by movement of the template during recall. In both cases, location in an extrinsic reference frame during presentation does not correspond with location in the same reference frame during recall. Consider now the hypothesis that the impairment seen in Experiment 1 was due to interference from tracking the template during the encoding of the sequence. According to this hypothesis, movement during output should lead to little or no impairment relative to the stationary control condition, since the encoding and rehearsal of the sequence took place before any movement of the template occurred.

\section{Method}

Participants. Twenty-four participants, 19 females and 5 males, were drawn from the same population as in Experiment 1 . Their mean age was 22.1 years $(S D=4.84)$. Fourteen were paid; the rest took part to satisfy a course requirement.

Materials. The templates and sequences were constructed randomly on each trial in exactly the same way as in Experiment 1.

Procedure. The experiment involved two conditions, a control condition and a movement condition, each of which was presented as a block of 20 trials using a sequence length of seven positions. Data from the first five trials were excluded as practice. The order in which the two conditions was completed was counterbalanced. In both conditions, the presentation of each sequence was identical to that of the control condition of Experiment 1, in which the template remained stationary in the center of the screen. The conditions differed only after the recall tone was sounded.

In the control condition, the display remained stationary in the center of the screen during recall of the spatial sequence. In the movement condition (Figure 1B), after the 0.5 -sec retention interval and the tone, the participant recalled the first square and used the mouse to click on it. The square was immediately filled, and at this point the mouse cursor disappeared, the template moved across the screen for $0.5 \mathrm{sec}$, and the cursor then reappeared. The participant then recalled the second square, and so on until seven squares had been chosen. The extent and speed of template movement after selection of each square were the same as the extent and speed of the movement used during presentation in the movement condition of Experiment 1. When seven squares had been selected, the screen was then cleared, and the participant clicked the mouse in the bottom right corner of the screen to initiate the next trial.

The experimental conditions were preceded by a short practice of eight trials in which a sequence of six locations was shown, under stationary conditions.

\section{Results and Discussion}

For each condition, recall accuracy at each serial position is shown in Figure 3. The level of performance is very similar in the two conditions. The accuracy data were analyzed by a $2 \times 2 \times 7$ split plot ANOVA, with the order of performing the conditions as a between-groups factor and with movement condition and serial position as the repeated measures. There was no significant main effect of order $[F(1,22)<1.0]$ or of movement condition $[F(1,22)<1.0]$. As expected, there was an effect of serial position $\left[F(3.4,75.9)=32.1, M S_{\mathrm{e}}=0.018, p<.001\right]$. There was also a significant three-way interaction between serial position, condition, and order $[F(3.2,70.7)=$
$3.9, p<.05]$. The three-way interaction can be summarized as follows. Performance in the control condition was better when the control condition occurred second, but the advantage appeared only toward the end of the list. The moving condition was advantaged when it occurred first, but only at the beginning of the list. This suggests that the moving condition promoted either a change in grouping strategy or a shift in attention from earlier to later serial positions.

Response accuracy was also assessed by measuring the proportion of correct trials. This measure gave a value of $.29(S D=.199)$ for the control condition and $.26(S D=$ .197) for the movement condition. Again, there was no significant difference in accuracy across the two conditions $[t(23)<1.0]$.

As explained above, if performance in the stationary condition of this task was supported by extrinsically coded memory for each location at the time of recall, then moving the template during recall should have decreased accuracy. This decrease was not observed. Thus spatial encoding of position with respect to an extrinsic frame of reference was not used at the time of recall. However, Experiment 1 showed a marked effect of template movement during input. Considering these two results together, one possible interpretation is that temporary knowledge of extrinsic position may be used to support the construction of a template-centered representation, which is used to recall the sequence. In this account, spatial span is essentially based on template-centered representations, but extrinsic representation that is sensitive to template movement provides an intermediate step. An alternative account is that extrinsic encoding of position does not take place at all, but movement during input disrupts template-centered encoding of position. By this account, it is difficult to encode a spatial sequence in a local frame of reference that is itself

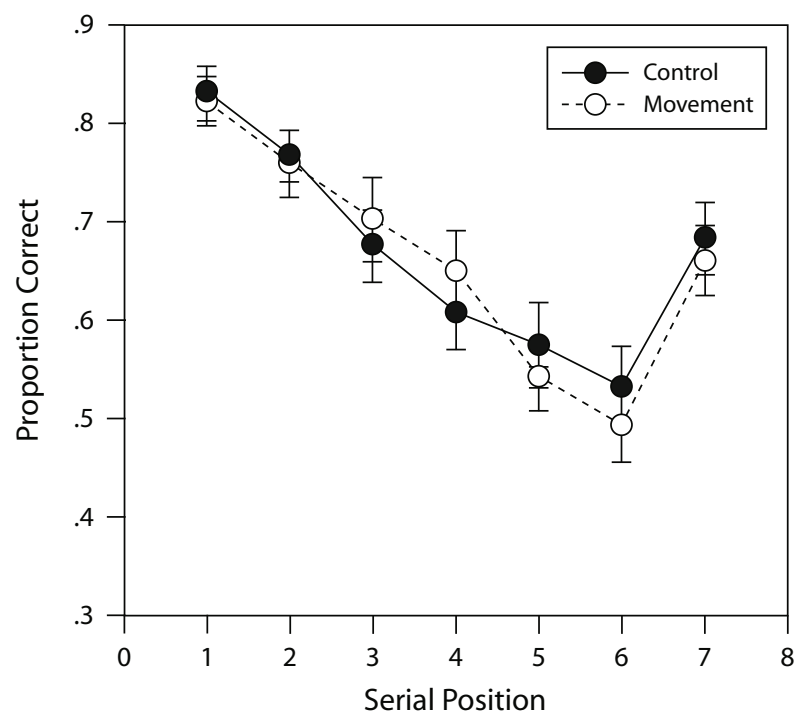

Figure 3. Accuracy in Experiment 2 of serial spatial recall in the control condition (Corsi template was stationary at presentation and during recall) and when the template moved relative to the observer during recall. 
moving unpredictably. Further studies are needed to determine the effect of concurrent movement on encoding. However, at the time of recall, spatial span appears to use only template-centered descriptions of spatial position that are resistant to translation movements. This could mean that in the control condition, positional information based on an extrinsic frame of reference is no longer available at the time of recall or that extrinsic spatial information is available, but it is redundant with template-centered information. If the information in extrinsic and template-centered frames of reference were redundant, then clearly the unavailability of extrinsic information in the experimental condition would not lead to a decrease in performance.

One way to resolve this ambiguity would be to set up conditions such that memory for extrinsically coded locations would be advantageous and memory for templatecentered locations would not be, or vice versa. To do this, we would take advantage of the fact that spatial span sequences are rapidly learned if repeated across trials, as in the Hebb effect (see, e.g., Gagnon et al., 2004). Moving the template appropriately can arrange sequences of either extrinsic or template-centered locations so that they are repeated across trials. If, at the end of a trial, memory for the sequence is encoded in both extrinsic and templatecentered descriptions, then learning should occur if either type of representation is repeated. If, on the other hand, memory for sequences is based solely on template-centered descriptions, then sequences repeated in the extrinsic reference frame should not be learned.

Experiments 3 and 4 examined the effect of repeating spatial sequences in the extrinsic and template-centered reference frames, respectively. In both experiments, the template moved in the same way during presentation and during recall; thus, within each trial, a correctly recalled square occupied the same extrinsic and template-centered location during presentation and during recall. In the experimental conditions, the same sequence of locations was repeated on each trial, either in the same extrinsic position or in the same template-centered position. We could then ask whether learning was facilitated if the sequence of locations with respect to an extrinsic frame of reference was repeated across trials while template-centered sequences varied across trials (Experiment 3). Experiment 4 examined the converse situation in which template-centered sequences were repeated across trials, whereas sequences in the extrinsic frame of reference varied.

\section{EXPERIMENT 3}

In Experiment 3, new random positions were chosen for the squares within the template on each trial (as they were for Experiments 1 and 2). However, the target locations on each trial were displayed at one of a small set of seven discrete extrinsic locations; that is, the filled squares indicating the spatial sequence were displayed using only seven fixed locations on the computer screen. The movements of the template were programmed so that in the constant condition, the sequence of seven extrinsic locations was identical on every trial, whereas in the variable condition, the seven extrinsic locations were displayed in a differ- ent order on every trial. To illustrate this another way, in the constant condition, the mouse movements required to recall the correct sequence were effectively the same on each trial, since the trajectory of target locations on the computer screen never varied. If memory for location in an extrinsic reference frame persisted throughout the trial, we would expect that repeatedly using the same extrinsic locations would lead to a gradual increase in performance across trials. However, if location with respect to an extrinsic reference frame was transient or not encoded in this task, there would be no gain across trials.

\section{Method}

Participants. Sixteen students at the University of Essex took part, of whom 5 were male and 11 were female. The mean age of participants was 22.4 years $(S D=2.6)$. None had participated in Experiments 1 or 2 .

Materials. To meet the requirements of the design, some features of the materials in the previous experiments were changed. Because the maximum amplitude of translation was now increased (for example, a square at the bottom right of the template might be displayed in a location toward the top left of the screen, and a square at the top left might be displayed the bottom right), the template was reduced in size. The size of the rectangular frame was reduced to $11.4 \times 8.3 \mathrm{~cm}$, and the size of the squares was correspondingly reduced to $0.9 \mathrm{~cm}$. As before, each square in the sequence was filled for $0.5 \mathrm{sec}$, followed by a 0.5 -sec interval during which all squares were unfilled. Since the duration of the movement was kept constant but the linear extent of movement varied greatly within a sequence, the speed with which the rectangular frame moved varied considerably. Between the display of successive squares, the movement of the frame had the potential to range from nil to almost twice the length of the frame diagonal $(25.6 \mathrm{~cm})$.

At the start of each condition of this experiment, a new set of seven, fixed-screen locations was selected randomly. This was done by generating a virtual template positioned in the center of the screen and then randomly selecting seven squares from it. The screen locations of these seven squares were the only positions at which the filled squares making up the spatial sequence were displayed.

Procedure. The sequence of events on each trial is illustrated in Figure 4. At the beginning of each trial, the template was shown in the center of the screen for $1 \mathrm{sec}$. Then, during the next $0.5 \mathrm{sec}$, it moved to a new position (Template Position 1), which was determined by the constraint that the first displayed square, at a randomly chosen position on the template, had to be displayed at one of the seven fixed screen locations. The template remained in the first position for $0.5 \mathrm{sec}$ while the first square of the sequence was displayed. The template then moved to a new position (Template Position 2) such that the second displayed square on the template was brought into correspondence with the second fixed-screen location, and so on until seven squares had been displayed. After the seventh square was displayed, the template moved back to the center of the screen over the next $0.5 \mathrm{sec}$ and remained there another $0.5 \mathrm{sec}$, after which a tone sounded. In the next $0.5 \mathrm{sec}$, the frame then moved to Screen Location 1 and remained there until the participant selected a square; it then moved to Screen Location $2(0.5 \mathrm{sec})$, where the second square was selected. This process continued until seven squares had been selected. At this point, the screen was cleared, and the participant clicked the mouse in the bottom right corner of the screen to initiate the next trial.

Each participant completed two conditions, each consisting of a block of 20 trials. In the constant condition (illustrated in Figure 4), the sequence of fixed-screen locations at which the squares were displayed was the same on all trials. In the other block (variable condition), seven fixed locations were used, but squares were displayed at these locations in a different random order on each trial. The order of conditions was counterbalanced across participants. In 
A

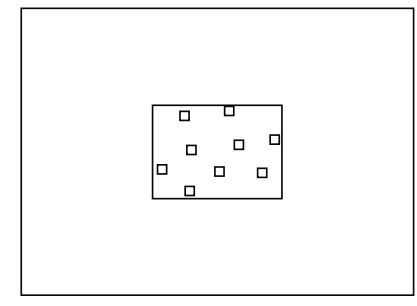

Start $1 \mathrm{sec}$
B
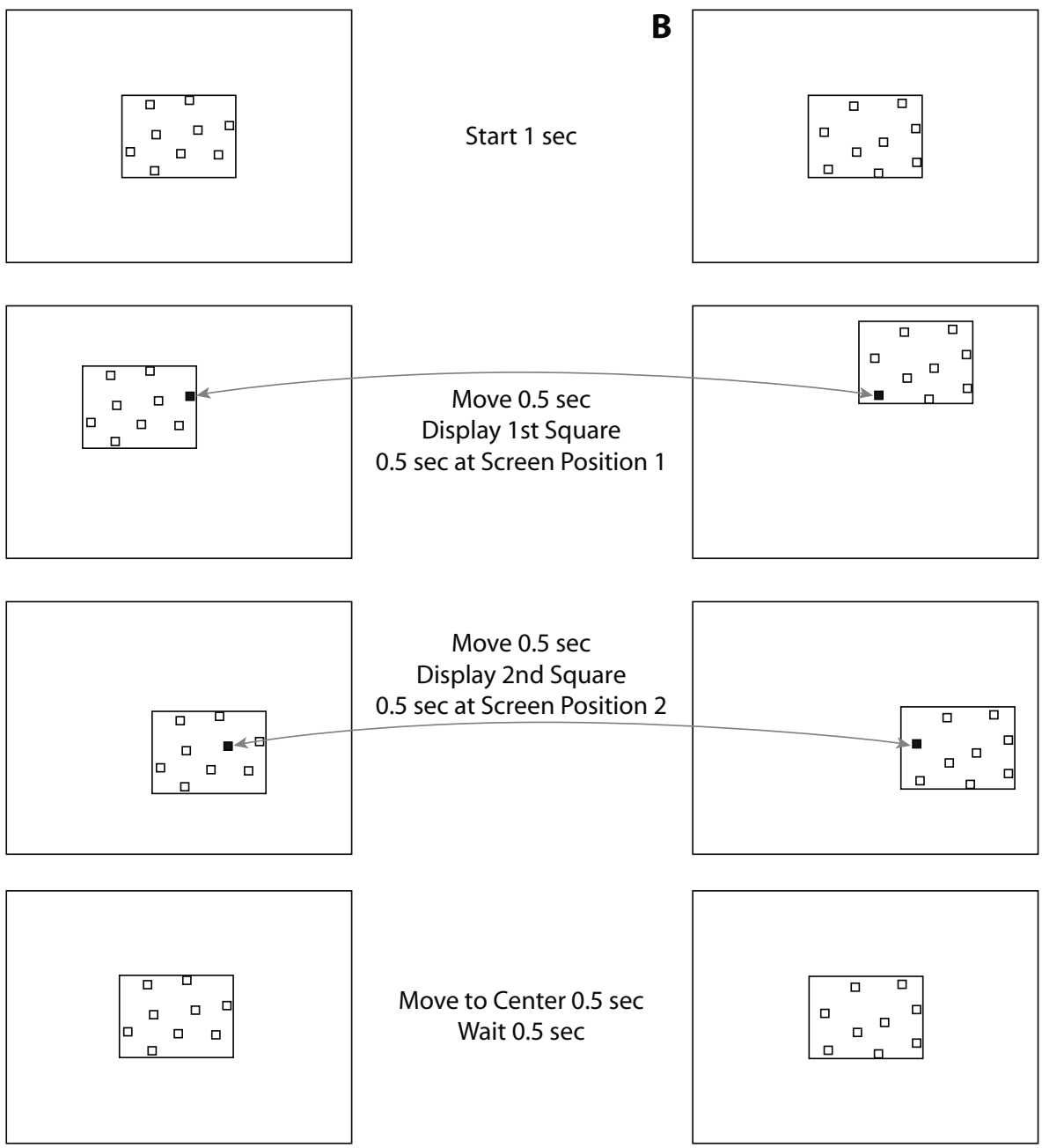

Move to Center $0.5 \mathrm{sec}$ Wait $0.5 \mathrm{sec}$

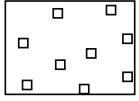

TONE
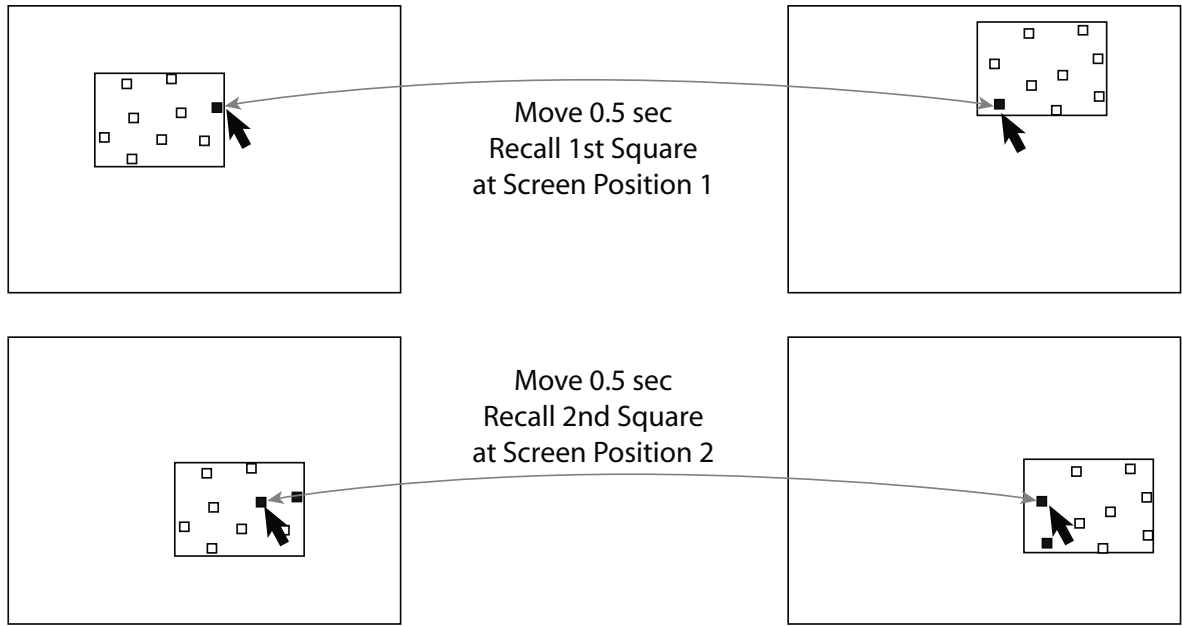

Figure 4. Panels A and B illustrate the sequence of displays in two trials from Experiment 3, constant condition. Pictures show the position of the template in relation to the border of the screen at the start of the trial, during the retention interval, and after each movement of the template, followed by the corresponding display or recall of each location. The template moves in the same way during input and during recall. The locations on the template change from trial to trial, but movements of the template are coordinated so that in the constant condition, the sequence of extrinsic locations is the same on each trial, as indicated by curved arrows. Bold arrows indicate the cursor position during recall. In this illustration, the sequence length is reduced to two locations. 
both conditions, the location of the squares on the template and the sequence in which the seven squares were displayed were chosen randomly on each trial. At the end of the experiment, participants were given a short questionnaire asking which of the two blocks was easier and if they had noticed any difference between the two experimental blocks of trials.

The experiment was preceded by two short practice sessions with sequence length of six. In the first session (six trials), the display was stationary during presentation and recall. In the second session (eight trials), the template moved to different randomly chosen template locations during presentation and to the same random locations during recall. As before, care was taken to ensure that participants remained still throughout the experiment, moving only the mouse during recall.

\section{Results and Discussion}

To show the effect of learning across trials, the mean proportion correct on each trial in the constant and variable conditions of this experiment is shown in Figure 5. Linear regression lines were fit to each curve, giving the following parameters; lower and upper 95\% confidence intervals are in parentheses. For the constant condition, the intercept was $.549(.480, .617)$, and the slope was $.0013(-.004, .007)$. For the variable condition, the intercept was $.582(.501, .662)$, and the slope was -.0021 $(-.009, .005)$. Clearly, performance across trials is very similar in the two conditions, and neither condition shows any evidence of learning across trials.

These data were further analyzed by a $2 \times 2 \times 20$ split plot ANOVA with order as the between-subjects variable and condition and trial number as the within-subjects variables. There was no main effect of order $[F(1,14)=$ $\left.1.27, M S_{\mathrm{e}}=0.594, p>.1\right]$ or any significant interaction of order with any other variable or combination of variables, so the data were pooled across the two orders. The resulting $2 \times 20$ repeated measures ANOVA showed no significant effect of condition $[F(1,15)<1.0]$. There was a nonsignificant effect of trial $\left[F(19,285)=1.52, M S_{\mathrm{e}}=\right.$ $0.062, p=.078]$. There was no interaction between trial and condition $\left[F(19,285)=1.26, M S_{\mathrm{e}}=0.069, p>.1\right]$. Again, these results suggest that having a constant sequence of extrinsic locations offers no advantage. This outcome would be expected if participants were encoding, remembering, and utilizing only the template-centered positions of the spatial sequence.

The questionnaire results were consistent. Of the 16 participants, 7 thought the constant condition was easier, 4 thought the variable condition was easier, and 5 thought they were equally difficult. Of those reporting that the constant condition seemed easier, no individual reported that the extrinsic locations were in the same invariant sequence.

The absence of learning across trials in the constant condition suggests that memory for extrinsically coded location did not persist from one trial to another and hence did not contribute to spatial learning across trials. This does not rule out a contribution of extrinsically coded memory to performance within a trial, although Experiment 2 suggests that it makes no independent contribution at the time of recall. We know that with stationary displays, Hebb learning (learning of sequences that are repeated on every third trial) readily occurs in spatial span (see, e.g., Gagnon

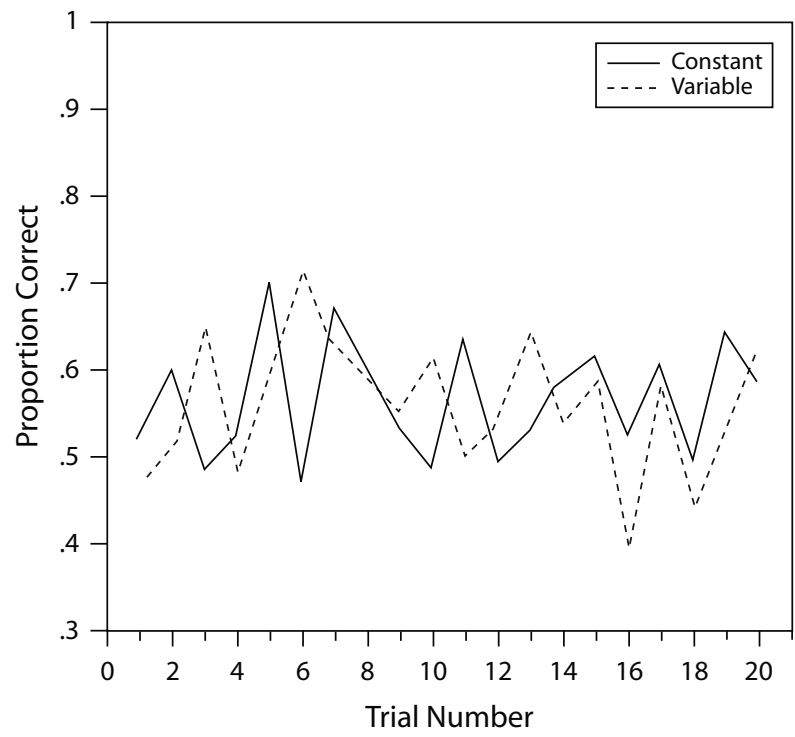

Figure 5. Accuracy of serial spatial recall when egocentric location was constrained (Experiment 3). Curves show performance across trials when sequences of extrinsic locations were repeated on each trial (constant condition) and when extrinsic sequences varied across trials.

et al., 2004). In this form, the same arrangement of squares is used on each trial, and a fixed sequence of squares is repeated so that template-centered coding is possible. The fact that there is no learning across trials for sequences of locations that are repeated in an extrinsic frame of reference (even if repeated on every trial) suggests that Hebb type learning normally occurs on template-centered spatial representations. However, Experiment 3 used an unusual form of presentation with unpredictable movements of the template at presentation and recall. It is possible that even repeated template-centered sequences would be difficult to learn under these conditions. In other words, the failure to demonstrate learning across trials might be a consequence of the moving template rather than the form of coding. To test this, Experiment 4 investigated learning of repeated template-centered sequences when the template moved during input and output.

\section{EXPERIMENT 4}

This experiment was the converse of Experiment 3. Here the extrinsic locations of the displayed sequences (i.e., position on screen or position with respect to the stationary observer) were novel and randomly selected on each trial, whereas the template-centered locations were fixed, like the blocks in the standard Corsi task. In one condition, the sequence of template-centered locations was constant across trials; in the other, it varied randomly across trials. If spatial locations are encoded primarily in template-centered coordinates, as the above experiments on the whole suggest, we should expect rapid and substantial learning in the constant condition when the templatecentered sequence is repeated across trials. 
In contrast to the previous experiments, in Experiment 4, one constant template was used throughout each block of trials. This template consisted of the rectangular frame plus one fixed arrangement of squares, generated randomly as described for Experiment 1. As in Experiment 3 , on each trial the rectangular frame and squares moved during input and output, and the extrinsic location (i.e., screen position) at which each square was displayed was the same in the presentation and recall phases. However, unlike in Experiment 3, these extrinsic locations were randomly chosen anew on each trial. There were two conditions. In the constant condition, the same sequence of template-centered locations was displayed on each trial. In the varied condition, a fixed subset of seven squares was chosen on which the sequence was displayed, but the squares were displayed in a new random order on each trial. If memory for the sequence of locations is retained in a template-centered framework, then we should expect rapid learning to occur across the constant block of trials, whereas performance should be relatively stable in the variable block of trials.

\section{Method \\ Participants. Eight students, 5 females and 3 males, at the Uni- versity of Essex participated. The mean age was 26.4 years $(S D=$ 6.4). Five were paid; the remainder participated as part of a course requirement. \\ Materials. The dimensions of the template were the same as in Experiment 3, but here only one template was used for each block of 20 trials. Thus, on each trial, the squares were in the same template- centered positions. In contrast to Experiment 3, on each trial in this experiment a new sequence of seven screen locations was chosen, corresponding to seven squares selected from a new random virtual template placed centrally on the screen. As before, the movement of the (now invariant) template was constrained so that each square that formed part of the spatial sequence on the template was displayed at each of the (new, randomly chosen) screen locations. As in Ex- periment 3, the same set of template movements was used during presentation and during recall so that correctly recalled squares oc- cupied the same extrinsic and template-centered positions at input and during recall.}

Procedure. The sequence of events on each trial is illustrated in Figure 6. As in Experiment 3, at the beginning of each trial, the template was shown in the center of the screen for $1 \mathrm{sec}$. Then, over the next $0.5 \mathrm{sec}$, it moved to a new position (Template Position 1), which was determined by the constraint that the first square had to be displayed at the first randomly chosen screen location. The template remained in this position for $0.5 \mathrm{sec}$ while the first square of the sequence was displayed. The template then moved to a new position (Template Position 2) such that the second displayed square on the template was brought into correspondence with the second screen location; this continued until seven squares had been displayed. After the seventh square was displayed, the template moved back to the center of the screen over the next $0.5 \mathrm{sec}$ and remained there for another $0.5 \mathrm{sec}$, after which a tone sounded. In the next $0.5 \mathrm{sec}$, the frame then moved to Screen Location 1, remained there until the participant selected a square, then moved $(0.5 \mathrm{sec})$ to Screen Location 2, where the second square was selected. This process continued until seven squares had been selected. At this point the screen was cleared, and the participant clicked the mouse in the bottom right corner of the screen to initiate the next trial.

Each participant completed two conditions, each consisting of a block of 20 trials. In the constant condition (illustrated in Figure 6), the sequence of template squares was the same on all trials. In the other block (variable condition), seven selected squares on the template were shown in a different random order on each trial. Performance in the variable condition was measured across a block of 20 trials. Because rapid learning was anticipated in the fixed condition, a block of up to 20 trials was allowed, but the trials ceased if four successive trials were completely correct, on the assumption that all subsequent trials would also be correct. The two conditions were run in a counterbalanced order. Otherwise, the timing of the displays, the practice procedures, and all other details matched those of Experiment 3.

\section{Results and Discussion}

The mean proportion correct on each trial for each condition is shown in Figure 7, which clearly shows a marked effect of learning in the constant condition relative to the variable condition. In most cases, memory for the spatial sequences in the constant condition rapidly approached ceiling, and only one participant required all 20 trials to learn the constant sequence. If the stopping rule was applied and the participant therefore completed fewer than 20 trials, we assumed that the participant's responses on all subsequent trials would have been correct. No participant completed four successive trials without error in the variable condition. The data were analyzed by a $2 \times 2 \times$ 20 split plot ANOVA with order as the between-subjects factor and condition and trial as the within-subjects factors. This showed no significant effect of order or any interactions involving order, so the orders were combined into a $2 \times 20$ repeated measures ANOVA. As expected, there were significant effects of condition $[F(1,7)=33.4$, $\left.M S_{\mathrm{e}}=.136, p<.001\right]$ and of trial $[F(5.26,36.8)=2.79$, $\left.M S_{\mathrm{e}}=.120, p<.05\right]$.

All participants correctly noticed that the sequences in the constant condition were the same and that this made these sequences easier to learn. Thus, in contrast to the previous experiment, in which repeating a sequence in an extrinsic frame of reference had no discernible effect and was not detected, in this experiment a repeated sequence in the template-centered frame of reference was rapidly learned across trials and was easily detected by observers.

\section{GENERAL DISCUSSION}

These four experiments have investigated the recall of spatial sequences displayed on a VDU screen. Each sequence consisted of squares displayed in seven locations, which were chosen from the nine squares displayed on the template. The experiments took place in an illuminated experimental booth so that the computer screen and the surrounding environment were visible at all times. All four experiments used smooth translation movements of the template to dissociate spatial position, as specified in two classes of reference frame. The first of these is the templatecentered frame of reference, which should be resistant to translation since the description is based on spatial relationships extracted from the template itself. The second type is less well defined but involves an extrinsic frame of reference, which may be egocentric (specifying position in relation to the body axis of the observer) or environmental (specifying position on the computer screen itself or in relation to the immediate surroundings). In either case, extrinsically coded positions are sensitive to translation, 
A

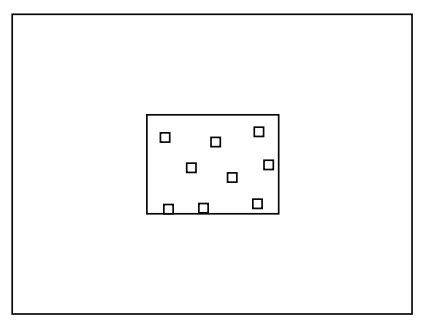

B
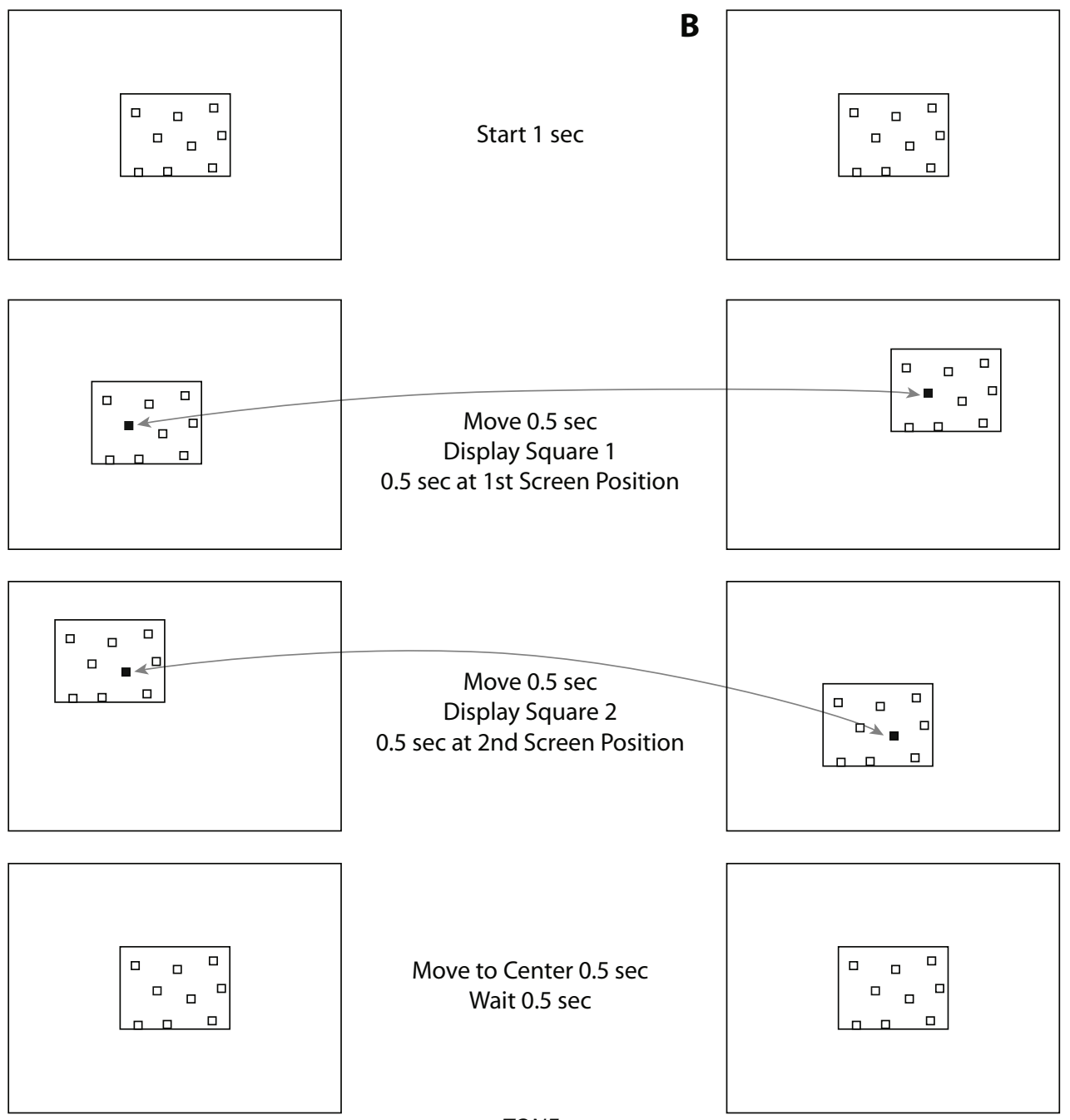

Move to Center $0.5 \mathrm{sec}$ Wait $0.5 \mathrm{sec}$

TONE
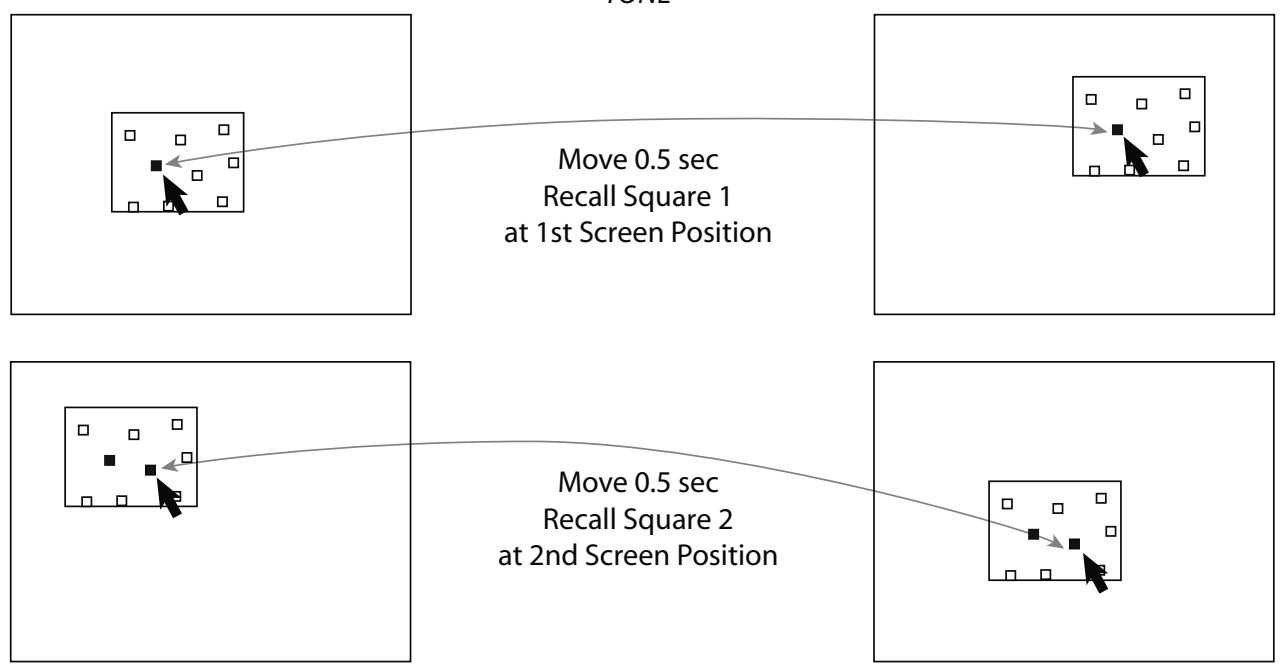

Figure 6. Panels A and B illustrate the sequence of displays in two trials from Experiment 4, constant condition. Pictures show the position of the template in relation to the border of the screen at the start of the trial, during the retention interval, and after each movement of the template, followed by the corresponding display or recall of each location. The template moved in the same way during input and during recall. The same sequence of squares (template-centered positions) was repeated on each trial. Movements of the template ensured that the sequence of extrinsic locations varied randomly from trial to trial, as indicated by curved arrows. Bold arrows indicate the cursor position during recall. In this illustration, the sequence length is reduced to two locations. 
which changes the spatial relationships between features of the template and the spatially fixed extrinsic reference positions of the observer or the local environment.

\section{Summary and Interpretation of Results}

The results show that translation of the template in between the display of each location during the presentation of a spatial sequence impaired performance (Experiment 1). In contrast, no performance impairment occurred if the template was moved during recall of a sequence that had been presented while the template was stationary (Experiment 2). Learning across trials occurred quickly if spatial sequences specified as template-centered locations were repeated (Experiment 4), but there was no evidence of learning across trials when extrinsic locations were held constant (Experiment 3).

Taken together, Experiments 2, 3, and 4 strongly endorse the position that under the conditions established in these three experiments, spatial span utilizes a templatecentered description for spatial positions rather than a description based on an extrinsic reference frame. When the extrinsic positions of targets were changed during recall relative to input positions (Experiment 2), spatial memory performance was unaffected. In Experiments 3 and 4, memory for extrinsic position was potentially available to recall the sequences because the template moved along identical trajectories during presentation and recall. However, repeating the same sequence of extrinsic locations on every trial did not enhance learning (Experiment 3), whereas when the same template-centered sequence was repeated across trials, learning was rapid and, in most cases, complete within a few trials (Experiment 4).

Previous studies of immediate recall of single spatial locations suggest that when local landmarks are visible, locations are encoded in relation to these landmarks, even though egocentric encoding is still possible (see, e.g., Diedrichsen et al., 2004; Lemay et al., 2004). Since in the present study the visual environment was visible at all times, we might expect egocentric encoding to have made little contribution to spatial span. The results from Experiments 2, 3, and 4 are fully consistent with this position, with the caveat that the egocentric reference frame cannot be isolated using the current paradigm.

The issue of spatial encoding when multiple reference frames are available was explored in the long-term memory studies of McNamara and colleagues (e.g., Mou et al., 2004; Roskos-Ewoldsen et al., 1998; Shelton \& McNamara, 2001). They asked participants to imagine a particular orientation with respect to a previously learned array and reported that the accuracy of making relative direction judgments was dependent on the imagined orientation. From this anisotropy, they inferred the orientation of the reference frame as represented in memory. Some studies using this technique showed that the memory representation was not necessarily determined by viewer position and that if several possible reference frames were available, then often one of these would predominate (McNamara, 2003; Roskos-Ewoldsen et al., 1998). Of critical relevance to the present study, some results indicated that participants used intrinsic reference frames based on the structure of the display if these frames facilitated encoding of spatial relations (Mou \& McNamara, 2002, Experiment 3). Such reference frames are analogous to the template-centered descriptions proposed here, except that they operated on a larger scale. Also, when proximal and more distant environmental reference frames were in conflict, the local reference frame predominated (Shelton \& McNamara, 2001, Experiment 3). The latter finding may indicate that proximal reference frames are used for the description of spatial relations wherever possible, which is again consistent with the use of template-centered encoding in the present experiments. The use of proximal reference frames should increase the precision of spatial localization if localization error depends on the distance to the nearest reference point.

Experiments 2, 3, and 4 provide considerable evidence in favor of template-centered spatial encoding. However, Experiment 1 showed a clear impairment caused by translation of the template during presentation of the sequence. As noted previously, this can be interpreted in two ways. The first explanation is that spatial position was encoded using an extrinsic reference frame that was sensitive to translation. However, since the recall phase was not affected by translation, we would have to assume that spatial position was initially encoded extrinsically and that this spatial description was then transformed into an intrinsic, template-based description before output. According to this account, spatial position was represented extrinsically in spatial span but only transiently during initial phases of the task. This account finds some resonance with two recent theories of spatial encoding, both of which propose that egocentric encoding of location is a transient phenomenon implicated in the control of action and in updating the relative positions of objects as the observer moves (Mou et al., 2004; Sholl \& Nolin, 1997).

An alternative explanation proposes that spatial position is encoded using only an intrinsic, template-centered

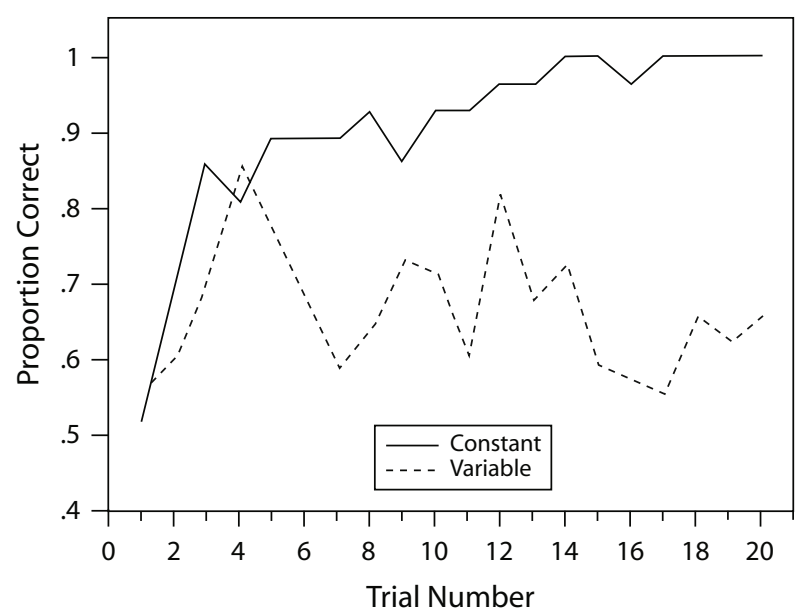

Figure 7. Accuracy of serial spatial recall when templatecentered location was constrained (Experiment 4). Curves show performance across trials when sequences of template-centered locations were repeated on each trial (constant condition) and when template-centered sequences varied across trials. 
reference frame but that the encoding process is itself impaired by template movement. This explanation receives considerable support from a long tradition of studies of interference in working memory. Spatial shifts of attention in response to auditory or visual targets, spatial tapping, and saccadic or pursuit eye movements are all known to impair spatial span during the retention interval (see, e.g., Pearson \& Sahraie, 2003; Smyth \& Scholey, 1994). Pearson and Sahraie reported that eye movement conditions gave greater interference than covert attention shifts to the same targets without eye movements and suggested that interference was specific to the oculomotor control system. The translation movements of the present studies seem to require pursuit eye movements to follow the template, although at present we have no eye movement data to support this. However, the present experiments differed from traditional dual task paradigms in two ways. First, the shifts of attention that followed the moving template were directed toward critical aspects of the display rather than to task-irrelevant, distracting stimuli. Second, in the present study the template moved only during input and/or during recall, with a short retention interval. If the account based on spatially directed attention is correct, then movement of the template during the retention interval should also impair performance by interfering with rehearsal, as in the earlier dual task studies. This prediction has yet to be tested.

To summarize, the conclusions from the present study are as follows. At the time of recall, memory for locations appears to be based on a translation-independent representation that is probably template based. But translation during presentation is disruptive. This may occur because template-based encoding is disrupted by attention shifts or by eye movements. Alternatively, translation during input may be disruptive because a translation-sensitive, possibly egocentric mechanism is required to encode the sequences. It is possible that these two explanations are related-if, for example, some form of egocentric coding is required to encode or rehearse the sequence of locations, which is in turn sensitive to attention shifts or oculomotor interference.

\section{Limitations of the Present Study}

The present study examined the effect of translating of the template in presentations of spatial span with a stationary observer. We have already noted that this method cannot distinguish between (1) extrinsic, egocentric reference frames that may be body or head centered and (2) various environmental reference frames based on features local to the display. However, the results suggest that extrinsic reference frames may play only a minor role in spatial span and do not contribute at all to learning across trials.

One possible account of template-centered encoding, not previously discussed, is that the positional code used in spatial span is retinotopic. With a stationary template, eye movements do not occur during encoding or during the retention interval of spatial span, and fixation of a stationary target does not impair performance (Pearson \& Sahraie, 2003). One potential strategy in the present study would be for participants to fixate one point on the template so that the locations would be described in an eyecentered or retinotopic reference frame. This explanation seems unlikely. Coding position relative to current gaze direction should be resistant to translation movements, provided that fixation is at the same position on the template at the time that each square is displayed. Thus, it is hard to see why this type of encoding would be sensitive to translation only at input. Although it is possible that different egocentric frames of reference are used at input and during recall, it seems unlikely that information initially encoded in a body- or head-centered frame would be recoded into a potentially less stable eye-centered reference frame during output.

With respect to the design of the movement conditions of Experiments 1 and 2, the sequence of template movements was generated in the same way at presentation (Experiment 1) and during recall (Experiment 2). This led to a small asymmetry in the spatial relationships between study and test positions. In Experiment 1, all serial positions were tested at a different extrinsic location from their positions at input, whereas in Experiment 2, Serial Position 1 was recalled at the same location, with the template in the center of the screen. This could potentially account for the present results if extrinsic encoding was critical for recalling only the first serial position and if all subsequent serial positions were specified in relation to the first using template-centered coordinates. Future experiments will address this issue and the more general question of whether spatial span is enhanced when the locations of a sequence are tested and recalled in the same extrinsic locations.

For convenience, the present study used a computer mouse to record responses. With this device, movement of the cursor on the screen differs in both direction and extent from movement of the controlling hand and the device. More evidence of extrinsic encoding might be found if direct pointing responses were made - through use of a touch screen, for example. However, in their studies of immediate recall of single locations, Diedrichsen and colleagues (Diedrichsen et al., 2004; Werner \& Diedrichsen, 2002) found similar patterns of landmark-based errors with direct and mouse-based responses, suggesting that mode of response did not influence the reference frame.

A related concern arising from Experiment 3 is that learning direct pointing movements across trials might be easier than learning mediated and rather noisy mousetracking movements. Although in principle the same mouse movements could be used to recall the correct sequence on each trial in the constant condition of Experiment 3, in practice, mouse movements were often fragmented and discontinuous. It remains to be seen if learning across trials would occur with direct pointing in this task.

Finally, it should be noted that the present experiments were conducted on a limited spatial scale, restricted by the size of the monitor used. It is possible that extrinsic coding of spatial location could be used on larger spatial scales. For example, egocentric coding based on body-centered coordinates might not have sufficient spatial resolution to specify targets on the small templates used here but might be useful on larger spatial scales. If so, evidence of extrinsic encoding might be found with larger display formats. It should be noted also that the templates used in Experiments 1 and 2 were larger than those used in the 
later experiments. It is possible that this change of scale may have discouraged the use of extrinsic encoding in these experiments. However, the use of intrinsic reference systems appears to operate over a range of spatial scales (see, e.g., Roskos-Ewoldsen et al., 1998).

\section{Implications for Spatial Span}

The results of these studies suggest that spatial span is based on an intrinsic, template-centered frame of reference. This we believe to be similar to the display-centered reference frame described by Mou \& McNamara (2002). The present study has not tested the effects of changing template orientation, but it is likely that the representation used in spatial span is orientation sensitive. All the evidence from related studies suggests that the relations between the squares of the template are coded with respect to the canonical vertical and horizontal directions, which are consistent with intrinsic and extrinsic environmental features such as the sides of the squares, the frame of the template, the edges of the computer screen, the walls of the room, the orientation of the observer (Shelton \& McNamara, 2001; Wang, 2003), and the position of the display in the vertical, frontal plane (Sholl \& Nolin, 1997).

The notion of space implied by the present study is important for our understanding of spatial span. Although some previous researchers have claimed that spatial span is encoded in an egocentric framework (Aguirre \& D'Esposito, 1999), our results suggest that in spatial span, the target squares are identified by local spatial relationships within the boundaries of the template (see also Kessels et al., 2000). Of course, spatial span is just one of a wide variety of spatial tasks, so this result cannot necessarily be generalized more widely.

For example, many studies of human and animal spatial cognition have addressed the ecologically important task of navigation through environments in which the task is to reach a target location from different starting positions. Many studies have examined the performance of humans on tasks devised to explore cognitive maps in animals. These studies have implicated hippocampal or right temporal lobe structures in neuropsychological cases (see, e.g., Aguirre \& D’Esposito, 1999; Holdstock et al., 1999; Holdstock et al., 2000; Morris \& Parslow, 2004) or neuroimaging data (see, e.g., Bohbot, Iaria, \& Petrides, 2004; Parslow et al., 2004). The intrinsic, templatecentered representation that appears to support spatial span would seem to be quite different from the viewpointindependent spatial descriptions that support navigation. From a neuropsychological perspective, spatial span is sensitive to parietal rather than temporal lesions (Corsi, 1972; Ferreira et al., 1998; Gagnon et al., 2004; Rausch \& Ary, 1990). Although it is tempting to attribute the neuropsychological dissociation to the demands posed by different spatial reference frames, other interpretations are possible.

Recent studies suggest that successful performance on spatial span depends on forming an efficient description of the sequence as a path or trajectory across locations (see, e.g., DeLillo, 2004; Kemps, 2001; Parmentier et al., 2005). Thus, the ability to individuate specific locations, which we propose occurs with respect to an intrinsic frame of reference, is necessary for spatial span but not sufficient to explain performance. Online processing, such as rehearsal of previous locations, may be necessary to describe a trajectory of locations, and Gestalt processes may act to code this path more efficiently. The main point here is that locations, trajectories, and patterns all have to be encoded with respect to a spatial frame of reference. The present evidence suggests that this spatial frame of reference is based on the template itself.

Finally, there may be subtle differences between contemporary versions of spatial span. In the current version and in the classical Corsi task, the surrounding frame and all the possible locations are continuously present. However, in the dots task developed by Jones and his colleagues (Jones, Farrand, Stuart, \& Morris, 1995; see also Parmentier et al., 2006), only one location is displayed at a time, along with the continuously present rectangular frame. Thus, for each location displayed, only the frame is available as a visible landmark. In terms of templatecentered encoding of position, this is quite a fundamental difference, and it is not clear why this does not make the task much harder. Memory for previous locations may be used as landmarks to encode new positions, or there may be greater involvement of extrinsic encoding. Although these different versions of spatial span appear to measure the same ability, they have not been compared directly. A further issue in this regard is that the reference frame may vary between $2-\mathrm{D}$ vertical, planar computer displays and real, 3-D displays, especially if the reference frame is observed from a low viewpoint (cf. Sholl \& Nolin, 1997).

\section{AUTHOR NOTE}

I thank Laura Carlson and three anonymous reviewers for helpful comments on an earlier draft. Correspondence concerning this article should be addressed to S. E. Avons, Department of Psychology, University of Essex, Wivenhoe Park, Colchester, Essex CO4 3SQ, England (e-mail: savons@essex.ac.uk).

\section{REFERENCES}

Abrahams, S., Morris, R. G., Polkey, C. E., Jarosz, J. M., Cox, T. C. S., Graves, M., \& Pickering, A. (1999). Hippocampal involvement in spatial and working memory: A structural MRI analysis of patients with unilateral mesial temporal lobe sclerosis. Brain \& Cognition, 41, 39-65.

Aguirre, G. K., \& D’Esposito, M. (1999). Topographical disorientation: A synthesis and taxonomy. Brain, 122, 1613-1628.

Baddeley, A. D. (1986). Working memory. Oxford: Oxford University Press.

Bohbot, V. D., Iaria, G., \& Petrides, M. (2004). Hippocampal function and spatial memory: Evidence from functional neuroimaging in healthy participants and performance of patients with medial temporal lobe resections. Neuropsychology, 18, 418-425.

Burgess, N., \& Hitch, G. J. (1999). Memory for serial order: A network model of the phonological loop and its timing. Psychological Review, 106, 551-581.

Corsi, P. (1972). Human memory and the medial temporal region of the brain. Unpublished $\mathrm{PhD}$ thesis: McGill University.

De Lillo, C. (2004). Imposing structure on a Corsi-type task: Evidence for hierarchical organization based on spatial proximity in serialspatial memory. Brain \& Cognition, 55, 415-426.

Diedrichsen, J., Werner, S., Schmidt, T., \& Trommershauser, J. (2004). Immediate spatial distortions of pointing movements induced by visual landmarks. Perception \& Psychophysics, 66, 89-103. 
Feigenbaum, J. D., \& Morris, R. G. (2004). Allocentric versus egocentric spatial memory after unilateral temporal lobectomy in humans. Neuropsychology, 18, 462-472.

Feigenbaum, J. D., \& Rolls, E. T. (1991). Allocentric and egocentric spatial information processing in the hippocampal formation of the behaving primate. Psychobiology, 19, 21-40.

Ferreira, C. T., Vérin, M., Pillon, B., Levy, R., Dubois, B., \& AGID, Y. (1998). Spatio-temporal working memory and frontal lesions in man. Cortex, 34, 83-98.

Fink, G. R., Marshall, J. C., Weiss, P. H., Stephan, T., Grefkes, C., ShaH, N. J., ET AL. (2003). Performing allocentric visuospatial judgments with induced distortion of the egocentric reference frame: An fMRI study with clinical implications. NeuroImage, 20, 1505-1517.

Gagnon, S., Foster, J. K., Turcotte, J., \& Jongenelis, S. (2004). Involvement of the hippocampus in implicit learning of supra-span sequences: The case of SJ. Cognitive Neuropsychology, 21, 867-882.

Goldstein, L. H., Canavan, A. G., \& Polkey, C. E. (1989). Cognitive mapping after unilateral temporal lobectomy. Neuropsychologia, 27, $167-177$

Hasselbach-Heitzeg, M. M., \& Reuter-Lorenz, P. A. (2002). Egocentric body-centered coordinates modulate visuomotor performance. Neuropsychologia, 40, 1822-1833.

Holdstock, J. S., Mayes, A. R., Cezayirli, E., Aggleton, J. P., \& RoBERTS, N. (1999). A comparison of egocentric and allocentric spatial memory in medial temporal lobe and Korsakoff amnesics. Cortex, 35, 479-501.

Holdstock, J. S., Mayes, A. R., Cezayirli, E., Isaac, C. L., Aggleton, J. P., \& ROBERTS, N. (2000). A comparison of egocentric and allocentric spatial memory in a patient with selective hippocampal damage. Neuropsychologia, 38, 410-425.

Jones, D., Farrand, P., Stuart, G., \& Morris, N. (1995). Functional equivalence of verbal and spatial information in serial short-term memory. Journal of Experimental Psychology: Learning, Memory, \& Cognition, 21, 1008-1018.

KemPs, E. (2001). Complexity effects in visuo-spatial working memory: Implications for the role of long-term memory. Memory, 9, 13-27.

Kessels, R. P. C., De HaAn, E. H. F., Kappelle, L. J., \& Postma, A. (2002). Selective impairments in spatial memory after ischaemic stroke. Journal of Clinical \& Experimental Neuropsychology, 24, 115-129.

Kessels, R. P. C., Kappelle, L. J., de Haan, E. H. F., \& Postma, A. (2002). Lateralization of spatial-memory processes: Evidence on spatial span, maze learning and memory for object locations. Neuropsychologia, 40, 1465-1473.

Kessels, R. P. C., Postma, A., Kappelle, L. J., \& de HaAn, E. H. F. (2000). Spatial memory impairment in patients after tumour resection: Evidence for a double dissociation. Journal of Neurology, Neurosurgery \& Psychiatry, 69, 389-391.

Lawrence, B. M., Myerson, J., Oonk, H. M., \& Abrams, R. A. (2001). The effects of eye and limb movements on working memory. Memory, 9, 433-444.

Lemay, M., Bertram, C. P., \& Stelmach, G. E. (2004). Pointing to an allocentric and egocentric remembered target. Motor Control, 8, 16-32.

Malhotra, P., Jäger, H. R., Parton, A., Greenwood, R., Playford, E. D., BRown, M. M., ET AL. (2005). Spatial working memory capacity in unilateral neglect. Brain, 128, 424-435.

McNamara, T. P. (2003). How are the locations of objects in the environment represented in memory? In C. Freksa, W. Brauer, C. Habel, $\&$ K. F. Wender (Eds.), Spatial cognition III: Routes and navigation, human memory and learning, spatial representation and spatial learning (pp. 174-191). Berlin: Springer.

Milner, A. D., \& Goodale, M. A. (1995). The visual brain in action. Oxford: Oxford University Press.

Milner, B. (1971). Interhemispheric differences in the localization of psychological processes in man. British Medical Bulletin, 27, 272-277.

Morris, R. G., Garrud, P., Rawlins, J. N., \& O’Keefe, J. (1982). Place navigation impaired in rats with hippocampal lesions. Nature, 297, 681-683.

Morris, R. G., \& PARsLow, D. (2004). Neurocognitive components of spatial memory. In G. L. Allen (Ed.), Human spatial memory: Remembering where (pp. 217-247). Mahwah, NJ: Erlbaum.
Mou, W., \& McNamara, T. P. (2002). Intrinsic frames of reference in spatial memory. Journal of Experimental Psychology: Learning, Memory, \& Cognition, 28, 162-170.

Mou, W., McNamara, T. P., Valiquette, C. M., \& Rump, B. (2004). Allocentric and egocentric updating of spatial memories. Journal of Experimental Psychology: Learning, Memory, \& Cognition, 30, 142-157.

Page, M. P. A., \& Norris, D. (1998). The primacy model: A new model of immediate serial recall. Psychological Review, 105, 761-781.

Parmentier, F. B. R., Andres, P., Elford, G., \& Jones, D. M. (2006). Organization of visuo-spatial serial memory: Interaction of temporal order with spatial and temporal grouping. Psychological Research, 70, 200-217.

Parmentier, F. B. R., Elford, G., \& Mayberry, M. T. (2005). Transitional information in spatial serial memory: Path characteristics affect recall performance. Journal of Experimental Psychology: Learning, Memory, \& Cognition, 31, 412-417.

Parslow, D. M., Rose, D., Brooks, B., Fleminger, S., Gray, J. A., GiAMPIETRO, V., ET AL. (2004). Allocentric spatial memory activation of the hippocampal formation measured with fMRI. Neuropsychology, 18, 450-461.

Pearson, D. G., \& Sahraie, A. (2003). Oculomotor control and the maintenance of spatially and temporally distributed events in visuospatial working memory. Quarterly Journal of Experimental Psychology, 56A, 1089-1111.

PELLI, D. G. (1997). The VideoToolbox software for visual psychophysics: Transforming numbers into movies. Spatial Vision, 10, 437-442.

Postma, A., Kessels, R. P. C., \& van Asselen, M. (2004). The neuropsychology of object-location memory. In G. L. Allen (Ed.), Human spatial memory: Remembering where (pp. 143-160). Mahwah, NJ: Erlbaum.

RausCh, R., \& ARY, C. M. (1990). Supraspan learning in patients with unilateral anterior temporal lobe resections. Neuropsychologia, 28, 111-120.

Roskos-Ewoldsen, B., McNamara, T. P., Shelton, A. L., \& Carr, W. (1998). Mental representations of large and small spatial layouts are orientation dependent. Journal of Experimental Psychology: Learning, Memory, \& Cognition, 24, 215-226.

Schumann-Hengsteler, R., Strobl, S., \& Zoelch, C. (2004). Temporal memory for locations: On the coding of spatiotemporal information in children and adults. In G. L. Allen (Ed.), Human spatial memory: Remembering where (pp. 101-124). Mahwah, NJ: Erlbaum.

Shelton, A. L., \& McNamara, T. P. (2001). Systems of spatial reference in human memory. Cognitive Psychology, 43, 274-310.

Sholl, M. J., \& Nolin, T. L. (1997). Orientation specificity in representations of place. Journal of Experimental Psychology: Learning, Memory, \& Cognition, 23, 1494-1507.

Sмутн, M. M. (1996). Interference with rehearsal in spatial working memory in the absence of eye movements. Quarterly Journal of Experimental Psychology, 49A, 940-949.

Smyth, M. M., Pearson, N. A., \& Pendleton, L. R. (1988). Movement and working memory: Patterns and positions in space. Quarterly Journal of Experimental Psychology, 40A, 497-514.

Sмyth, M. M., \& Scholey, K. A. (1994). Interference in immediate spatial memory. Memory \& Cognition, 22, 1-13.

Stark, M., Coslett, H. B., \& Saffran, E. M. (1996). Impairment of an egocentric map of locations: Implications for perception and action. Cognitive Neuropsychology, 13, 481-523.

WANG, R. F. (2003). Spatial representations and spatial updating. In D. E. Irwin and B. H. Ross (Eds.), The psychology of learning and motivation: Vol. 42. Cognitive vision (pp. 109-156). San Diego: Academic Press.

Werner, S., \& DiEDrichSEN, J. (2002). The time course of spatial memory distortions. Memory \& Cognition, 30, 718-730.

Woodin, M. E., \& Allport, A. (1998). Independent reference frames in human spatial memory: Body-centered \& environment-centered coding in near and far space. Memory \& Cognition, 26, 1109-1116.

(Manuscript received April 29, 2005; revision accepted for publication January 22, 2006.) 